\title{
Detection of Tax-specific CTLs in lymph nodes of adult T-cell leukemia/lymphoma patients and its association with Foxp3 positivity of regulatory T-cell function
}

\author{
AYAKO ICHIKAWA $^{1}$, HIROAKI MIYOSHI ${ }^{1}$, FUMIKO ARAKAWA $^{1}$, JUNICHI KIYASU $^{1}$, \\ KENSAKU SATO $^{2}$, DAISUKE NIINO ${ }^{1}$, YOSHIZO KIMURA ${ }^{1}$, MAKI YOSHIDA ${ }^{1}$, \\ RIKO KAWANO $^{1}$, HIROKO MUTA ${ }^{1}$, YASUO SUGITA ${ }^{1}$ and KOICHI OHSHIMA ${ }^{1}$ \\ ${ }^{1}$ Department of Pathology; ${ }^{2}$ Biostatistics Center, School of Medicine, Kurume University, Kurume, Fukuoka 830 0011, Japan
}

Received May 12,2015; Accepted September 27, 2016

DOI: $10.3892 / \mathrm{ol} .2017 .6067$

\begin{abstract}
Human T-cell lymphotropic virus type (HTLV)-1 Tax is a viral protein that has been reported to be important in the proliferation of adult T-cell leukemia/lymphoma (ATLL) cells and to be a target of HTLV-1-specific cytotoxic T lymphocytes (CTLs). However, it is not clear how Tax-specific CTLs behave in lymph nodes of ATLL patients. The present study analyzed the immunostaining of Tax-specific CTLs. Furthermore, ATLL tumor cells are known to be positive for forkhead box P3 (Foxp3)and to have a regulatory T (Treg)-cell-like function. The association between T-reg function and number and activity of Tax-specific CTLs was also investigated. A total of 15 ATLL lymphoma cases with human leukocyte antigen (HLA)-A24, for which Tax has a high affinity, were selected from the files of the Department of Pathology, School of Medicine, Kurume University (Kurume, Japan) using a polymerase chain reaction (PCR) method. Immunostaining was performed for cluster of differentiation (CD) 20 , CD3, CD4, CD8, T-cell intracellular antigen-1 and Foxp3 in paraffin sections, and for Tax, interferon $\gamma$ and HLA-A24 in frozen sections. In addition, the staining of Tax-specific CTLs (HLA-A24-restricted) was analyzed by MHC Dextramer ${ }^{\circledR}$ assay in frozen sections. In addition, the messenger RNA expression of Tax and HTLV-1 basic leucine zipper factor were also evaluated by reverse transcription-PCR. Immunohistochemical staining of Tax protein in lymphoma tissue revealed the presence of positive lymphoma cells ranging from 5 to $80 \%$, and immunohistochemical staining of HLA-A24 revealed the presence of positive lymphoma cells ranging from 1 to $95 \%$. The expression of Tax and HLA-A24 was downregulated by
\end{abstract}

Correspondence to: Dr Ayako Ichikawa, Department of Pathology, School of Medicine, Kurume University, 67 Asahi-machi, Kurume, Fukuoka 830 0011, Japan

E-mail: rikoayako@hotmail.co.jp

Key words: human T-cell lymphotropic virus, adult T-cell leukemia/lymphoma, Tax-specific cytotoxic T-cell, regulatory T-cell, Foxp3 viral function. Foxp3, a marker for Treg cells, was expressed in $0-90 \%$ of cells. Several cases exhibited Tax-specific CTL (HLA-A24-restricted)-positive cells, and there was an inverse correlation between Tax-specific CTLs and Foxp3. However, neither Tax nor HLA-A24 expression was associated with CTL or Foxp3. Our study indicated the possibility that ATLL cells, which expressed Tax, target of CTL, evade the CTL-mediated immune control by expression of Foxp3 as a Treg function.

\section{Introduction}

Human T-cell lymphotropic virus type (HTLV)-1 is a retrovirus known to cause human infections, and it was first isolated from a patient with cutaneous T-cell lymphoma (1).

Although the majority of infected individuals remain lifelong asymptomatic carriers of the virus, $\sim 4 \%$ of seropositive individuals develop adult T-cell leukemia/lymphoma (ATLL) (2). ATLL is highly endemic, particularly in Southern Japan and in the Caribbean Basin, and exhibits rapid progression, drug resistance and poor prognosis $(3,4)$. ATLL patients are in a severely immunocompromised state, which leads to frequent and severe infectious complications, and the underlying mechanisms responsible for this remain unclear (5). Recently, several investigators have reported that forkhead box P3 (Foxp3), which is a master control gene for the development and function of cluster of differentiation (CD) 4+CD25+ naturally occurring regulatory $\mathrm{T}$ (Treg) cells, is expressed in the tumor cells of ATLL patients (6). The suppression of the host's normal effector T-cells [including cytotoxic T lymphocytes (CTLs)] by the tumor cells could result in a severely immunocompromised state, which is one of the clinical characteristics of ATLL patients (7). It was reported that HTLV-1 infection was associated with other infectious diseases such as Pneumocystis carinii pneumonia and strongyloidiasis (8). In these diseases, an increase in the Treg-cell number (9) and a decrease in the T helper 2 response (10) were observed. In addition, it can be envisaged that ATLL cells could function as Treg cells and lead to a profound immunosuppressive environment, enabling them to escape from the host immune response. The molecular mechanism by which Treg cells exert their suppressor/regulatory 
activity was regarded to require cell-to-cell contact with the cell being suppressed (11).

Tax, a viral protein, has been reported to be important in the proliferation of ATLL cells and a target of Tax-specific CTLs (12). Tax is encoded by the $\mathrm{pX}$ region between envelope and 3'-long terminal repeat (LTR) (13). Tax is considered to play a central role in ATL lymphomagenesis by its pleiotropic actions, including trans-activation of cell proliferation factors such as nuclear factor $-\kappa \mathrm{B}$, cAMP response element binding and the serum response factor pathway (14), and functional inactivation of cell cycle regulators such as p16, p53 and mitotic arrest deficient like 1 (15). Development of leukemia and lymphoma in mice transgenic for Tax was reported (16).

Tax is known to be a major target antigen of HTLV-1-specific CTLs (17). Kannagi et al (17) indicated that Tax-specific CTLs in ATLL patients are inactive, and that Tax-specific CTL response is strongly activated following hematopoietic stem cell transplantation (HSCT) in certain ATLL patients in long-term remission. These findings suggest that ATLL cells escape from the host immune system, and that reactivation of Tax-specific CTLs may provide promising prophylactic and therapeutic approaches for HTLV-1 carriers and for ATLL patients whose ATLL cells retain the ability to express Tax.

In addition, ATLL cells often contain genetic and epigenetic alterations of the 5'-LTR of the HTLV-1 provirus, resulting in the loss of Tax expression (18). Takeda et al (18) reported that Tax transcripts were detected in only $40 \%$ of all ATLL cases. Furthermore, human leukocyte antigen (HLA) class I antigen downregulation or loss has been detected in numerous malignancies, including melanoma, colon cancer, prostate cancer and lung cancer (19). This downregulation was not reported in ATLL, although it may cause ATLL cells to escape from the host immune system.

There were several reports on whether ATLL cells function as Treg cells in a peripheral blood autologous setting $(6,7,20,21)$, but it is not clear how Tax-specific CTLs behave in lymph nodes of ATLL patients, or whether ATLL cells in lymph nodes function as Treg cells.

In the present study, Tax-specific CTLs (HLA-A24-restricted) were stained using MHC Dextramer ${ }^{\circledR}$ assay. In addition, Tax, interferon (IFN) $\gamma$ and HLA-A24 were immunostained in frozen sections, and the association between Tax-specific CTLs and Tax expression, Foxp3 positivity and HLA-A24 expression was investigated in order to reveal the function of ATLL cells and Tax-specific CTLs in lymph nodes.

\section{Materials and methods}

Case selection. A total of 15 ATLL cases with HLA-A24 (for which Tax has a high affinity) were selected from the files of the Department of Pathology, School of Medicine, Kurume University (Kurume, Japan). The 15 patients were diagnosed between April 2004 and March 2012. All patients were positive for ATLA or amplified HTLV-1 pX gene. Patients lymph node samples were positive for HLA-A24, as detected by polymerase chain reaction (PCR).

Clinical information was obtained from clinical charts and attending physicians. Histologic review was conducted by three of the current authors (K.O., D.N. and A.I.), and lesions were classified into three types: i) Pleomorphic cell variant, large-sized cells predominant (pleomorphic large); ii) pleomorphic cell variant, medium-sized cells predominant (pleomorphic medium); and iii) anaplastic large cell variant (ALCL). The diagnosis was made as previously described (6), mainly by following the World Health Organization classification criteria (5).

The present study was approved by the Kurume University Institutional Review Board (Kurume, Japan), and was conducted in accordance with the ethical guidelines mandated by the Declaration of Helsinki.

Polymerase chain reaction (PCR) for the detection of HLA-A24. DNA samples were extracted using a commercial kit (Blood \& Tissue Genomic Extraction Miniprep System; Viogene BioTek Corp., Sunnyvale, CA, USA). PCR amplification was performed with AmpliTaq Gold Master Mix (Applied Biosystems; Thermo Fisher Scientific, Inc., Waltham, MA, USA).

HLA-A belongs to the HLA class I heavy chain paralogues. This class I molecule is a heterodimer consisting of a heavy chain and a light chain ( $\beta-2$ microglobulin). The heavy chain is $\sim 45 \mathrm{kDa}$ and its gene contains 8 exons. Polymorphisms within exon 2 and exon 3 are responsible for the peptide invading specificity of each class one molecule (22).

If exon 2 and exon 3 were detected by PCR, the cases were regarded as positive for HLA-A24. Exon 2 was amplified by semi-nested PCR using the sense primers 5'EX2-A (1st FW primer) for first-round PCR, and A24/EX2-FW1 (2nd FW primer) for second-round PCR, and anti-sense primer A24/EX2-RV2 (1st 2nd RV primer). Exon 3 was amplified by semi-nested PCR using the sense primer A24/EX3-FW4 (1st 2nd FW primer) and anti-sense primer 3'EX3-A (1st RV primer) for first-round PCR, and 3'EX3-R4 (2nd RV primer) for second-round PCR.

The first-round PCR conditions were as follows: Initial denaturation at $95^{\circ} \mathrm{C}$ for $10 \mathrm{~min}$, followed by 30 cycles of $95^{\circ} \mathrm{C}$ for $30 \mathrm{sec}, 60^{\circ} \mathrm{C}$ for $30 \mathrm{sec}$ and $72^{\circ} \mathrm{C}$ for $30 \mathrm{sec}$, with a final extension at $72^{\circ} \mathrm{C}$ for $10 \mathrm{~min}$. The second-round PCR conditions were as follows: Initial denaturation at $95^{\circ} \mathrm{C}$ for $10 \mathrm{~min}$, followed by 40 cycles of $95^{\circ} \mathrm{C}$ for $30 \mathrm{sec}, 60^{\circ} \mathrm{C}$ for $30 \mathrm{sec}$ and $72^{\circ} \mathrm{C}$ for $30 \mathrm{sec}$, with a final extension at $72^{\circ} \mathrm{C}$ for $10 \mathrm{~min}$. The primer sequences were as follows: 5'EX2-A, 5'-CCCCAGGCT CYCACTCCATGAGGTATTTC-3'; A24/EX2-FW1, 5'-CCA CTCCATGAGGTATTTCTC-3'; A24/EX2-RV2, 5'-GTTGTA GTAGCGGAGCGC-3'; A24/EX3-FW4, 5'-CACACCCTC CAGATGATGTT-3'; 3'EX3-A, 5'-TCTCCTTCCCGTTCT CCAGGTATC-3'; and 3'EX3-R4, 5'-CCCGTTCTCCAGGTA TCT-3'. The amplified PCR products were electrophoresed in $3 \%$ agarose gels and visualized by ethidium bromide staining under ultraviolet light. The PCR products were of the appropriate lengths of $\sim 250 \mathrm{bp}$.

Immunohistochemistry. Immunohistochemical staining for CD3, CD20, CD4, CD8, CD15, CD30, T-cell intracellular antigen (TIA)-1 and Foxp3 was performed using 2.5- $\mu$ m-thick, formalin-fixed, paraffin-embedded tissue sections. The slides were deparaffinized with xylene, followed by ethanol. Following rehydration with water, antigen retrieval was performed with EDTA or TE buffer in a microwave oven. Following cooling and rinsing with buffer, endogenous peroxidase activity was blocked by incubating in $3 \%$ hydrogen peroxide for $5 \mathrm{~min}$. Slides were incubated with monoclonal antibodies for $30 \mathrm{~min}$ 
at room temperature. The slides were incubated with an EnVision+ System horseradish peroxide-labeled anti-rabbit polymer (K4003; Dako, Glostrup, Denmark) for $30 \mathrm{~min}$ for CD4, and an EnVision+ System horseradish peroxide-labeled anti-mouse polymer for CD3, CD20, CD8, CD15, CD30, TIA-1 and Foxp3.

A portion of each sample was maintained at $-80^{\circ} \mathrm{C}$. These $2.5-\mu \mathrm{m}$-thick, fixed in cold acetone, frozen sections were examined using monoclonal antibodies against Tax, INF $\gamma$ and HLA-24. Following rehydration with water, endogenous peroxidase activity was blocked by incubating in $3 \%$ hydrogen peroxide for $5 \mathrm{~min}$. Slides were incubated with monoclonal antibodies for $30 \mathrm{~min}$ at room temperature. The slides were incubated with an EnVision+ System horseradish peroxide-labeled anti-rabbit polymer for $30 \mathrm{~min}$.

Visualization of immunostaining was performed using diaminobenzidine for $10 \mathrm{~min}$. Slides were counterstained with hematoxylin, dehydrated with ethanol and mounted under coverslips.

The antibodies used included anti-CD3 (clone F7.2.38; Dako; diluted, 1:50), anti-CD20 (clone L-26; Dako; diluted, 1:5) anti-CD4 (clone 4H5; Medical \& Biological Laboratories, Co., Ltd., Nagoya, Japan; diluted, 1:30), anti-CD8 (clone 1A5; Leica Microsystems GmbH, Wetzlar, Germany; diluted, 1:50), anti-CD15 (clone MMA; BD Biosciences, Franklin Lakes, NJ, USA; diluted, 1:50), anti-CD30 (clone Ber-H2; Dako; diluted, 1:100), anti-TIA-1 (clone 2G9A10F5; Immunotech; Beckman Coulter, Inc., Brea, CA, USA; diluted, 1:200) and anti-Foxp3 (clone FJK-16s; eBioscience, Inc., San Diego, CA, USA; diluted, 1:50). A portion of each sample was maintained at $-80^{\circ} \mathrm{C}$ in a deep freezer. These frozen tissues were examined using monoclonal antibodies against Tax (kindly donated by Dr M. Matsuoka; Kyoto University, Kyoto, Japan; diluted 1:200), INF $\gamma$ (ab9657; Abcam, Cambridge, UK; diluted, 1:1,000) and HLA-A24 (clone 17A10; MBL International Co., Woburn, MA, USA; diluted, 1:1,000).

Reverse transcription (RT)-PCR. Previous studies have reported that the Tax gene is expressed in few ATLL cases; however, the HBZ gene is expressed in all ATLL cases $(18,23)$ We evaluated the messenger RNA expression of tax and HBZ by RT-PCR in order to ascertain HTLV-1 infection. Complementary (c) DNA was reverse transcribed from $\sim 4.5 \mu \mathrm{g}$ total RNA using Ready-To-Go You-Prime First-Strand Beads (GE Healthcare Life Sciences, Chalfont, UK) and primed with an oligo(dT) oligonucleotide (GE Healthcare Life Sciences). cDNA ( $3 \mu \mathrm{l})$ was subjected to PCR using AmpliTaq Gold DNA Polymerase (Applied Biosystems; Thermo Fisher Scientific, Inc.). The oligonucleotide primers were as follows: Hemoglobin subunit zeta (HBZ) 5'-AAACGCATCGTGATCGGC AGCGAC-3' (sense) and 5'-CTTCCAACTGTCTAGTATAGC CATCA-3' (antisense); Tax, 5'-CTCTGGGGGACTATGTTC GGCC-3' (sense) and 5'-GTACATGCAGACAACGGAGCC T-3' (antisense); and $\beta$-actin, 5'-CAAGAGATGGCCACGGCT GCT-3' (sense) and 5'-TCCTTCTGCATCCTGTCGGCA-3' (antisense). Product sizes were 294 bp for HBZ, 373 bp for Tax and $275 \mathrm{bp}$ for $\beta$-actin. Amplification conditions consisted of denaturation at $95^{\circ} \mathrm{C}$ for $30 \mathrm{sec}(10 \mathrm{~min}$ for the first cycle), annealing at $60^{\circ} \mathrm{C}$ for $30 \mathrm{sec}$ and extension at $72^{\circ} \mathrm{C}$ for $1 \mathrm{~min}$ (10 min for the last cycle) for 35 cycles for HBZ and Tax, and 30 cycles for $\beta$-actin. The amplified products were evaluated in
$2 \%$ agarose gels and visualized by ethidium bromide staining under ultraviolet light. The quality of cDNA was monitored using RT-PCR with $\beta$-actin primers. cDNAs yielding a 275-bp product for $\beta$-actin messenger RNA without contamination with the 370-bp genomic amplification product were used for this experiment.

The following cell lines were used: MT-4, HTLV-1 infected cell line, as a positive control and Jurkat, T-cell acute lymphoblastic leukemia cell line (HTLV-1 uninfected cell line), as a negative control.

Detection of Tax-specific CTLs. The presence of HLA-A24/Tax reactive CD8+ T lymphocytes among infiltrating lymphocytes in lymph nodes of ATLL patients was evaluated by means of HLA/peptide dextramer staining. Fluorescein isothiocyanate (FITC)-conjugated HLA-A24/Tax dextramers (Immudex, Copenhagen, Denmark) were used to stain acetone-fixed, frozen materials as described previously $(24,25)$, and antigen-specific cells were visualized using a confocal laser microscope.

For staining with FITC-conjugated multimeric peptide/major histocompatibility complex (MHC) complexes, sections were cut (of 2.5- $\mu \mathrm{m}$ thickness) and air-dried for $30 \mathrm{~min}$ prior to be fixed in cold acetone for $5 \mathrm{~min}$. All the incubation steps were performed at room temperature in the dark, as follows: i) 45 min with the primary antibody (anti-CD8) (1:100 diluted); ii) cyanine 3-conjugated goat anti-mouse (1:500 diluted; 115-165-100; Jackson ImmunoResearch Europe, Ltd., Newmarket, UK) for $45 \mathrm{~min}$; and iii) dextramer for $75 \mathrm{~min}$. Between each step, the slides were washed three times for $5 \mathrm{~min}$ in PBS containing $0.1 \%$ bovine serum albumin. The slides were mounted in VECTASHIELD (Vector Laboratories, Inc., Burlingame, CA, USA) and kept in the refrigerator until observed under a fluorescence microscope (model BZ-9000; Keyence Corporation, Osaka, Japan).

Statistical analysis. Student's $t$ test and $\chi^{2}$ test were used for comparisons of clinical and pathological findings among different groups. $\mathrm{P}<0.05$ was considered to indicate a statistically significant difference.

\section{Results}

Histopathological and immunohistochemical features. The pathological findings are listed in Table I. The 15 cases enrolled in our study comprised 8 females and 7 males aged 55-83 years, with a mean age of 70 years. Of the 15 cases, 8 cases were classified as pleomorphic large, 6 cases as pleomorphic medium and 1 case as ALCL.

Immunohistochemical staining of CD3 demonstrated various lymphoma cells to be positive, ranging from 0 to $100 \%$ (median, 70\%). The number of CD4-positive lymphoma cells ranged from 60 to $100 \%$ (median, $100 \%$ ), while the number of CD8-positive lymphoma cells ranged from 0 to $100 \%$ (median, $0 \%$ ). A certain number of reactive lymphocytes in the background were also positive for CD8.

Fluorescent staining of Tax-specific CTLs and immunostaining of Foxp3. Among the 15 cases, there were positive Tax-specific CTLs in 9 cases (Fig. 1). The number of Tax-specific CTLs ranged from 0 to 8 cells $/ \mathrm{mm}^{2}$ (Table I). It should be noted 
Table I. Pathological findings of 15 cases.

Immunostaining

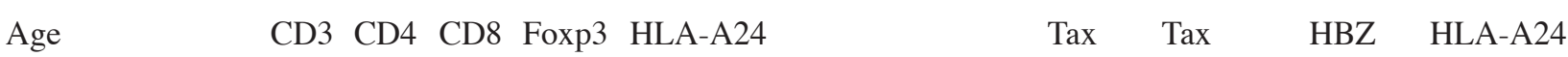

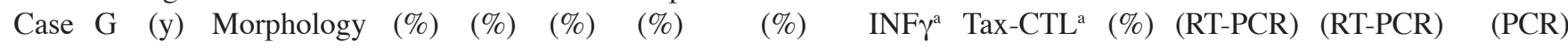

\begin{tabular}{rcccrrrrrrrrrr}
\hline 1 & F & 69 & Medium & 90 & 100 & 0 & 90 & 95 & 0.0 & 0 & 14 & $1+$ & $1+$ \\
2 & M & 62 & Medium & 70 & 60 & 70 & 50 & 95 & 1.0 & 0 & 9 & n.d. & n.d. \\
3 & F & 76 & Medium & 90 & 100 & 0 & 40 & 0 & 0.0 & 0 & 18 & $1+$ & $1+$ \\
4 & M & 75 & Medium & 90 & 100 & 90 & 40 & 10 & 0.5 & 0 & 6 & $1+$ & $1+$ \\
5 & F & 72 & Medium & 25 & 100 & 0 & 20 & 95 & 0.0 & 6 & 18 & $1+$ & $1+$ \\
6 & F & 71 & Medium & 50 & 100 & 0 & 5 & 10 & 0.5 & 6 & 6 & $1+$ & $2+$ \\
7 & M & 76 & Large & 34 & 100 & 0 & 10 & 50 & 0.5 & 4 & 10 & $1+$ & $2+$ \\
8 & M & 73 & Large & 100 & 100 & 90 & 10 & 80 & 1.0 & 2 & 18 & $1+$ & $2+$ \\
9 & F & 72 & Large & 100 & 100 & 100 & 10 & 10 & 1.0 & 4 & 2 & $2+$ & $2+$ \\
10 & M & 61 & Large & 5 & 100 & 0 & 7 & 80 & 0.5 & 7 & 4 & $2+$ & $2+$ \\
11 & F & 83 & Large & 70 & 100 & 0 & 5 & 80 & 1.0 & n.d. & 8 & $1+$ & $2+$ \\
12 & M & 55 & Large & 24 & 90 & 80 & 2 & 95 & 0.5 & 4 & 6 & $1+$ & $2+$ \\
13 & F & 80 & Large & 0 & 100 & 0 & 2 & 90 & 2.5 & 0 & 2 & $2+$ & + \\
14 & F & 66 & Large & 50 & 100 & 0 & 0 & 90 & 0.5 & 8 & 3 & $2+$ & + \\
15 & M & 69 & Anaplastic & 10 & 100 & 0 & 7 & 5 & 3.5 & 5 & 12 & $2+$ & + \\
\end{tabular}

${ }^{a}$ Levels are expressed as cells/mm². G, gender; F, female; M, male; y, years; CD, cluster of differentiation; Foxp3, forkhead box P3; HLA, human leukocyte antigen; INF, interferon; CTL, cytotoxic T lymphocyte; RT-PCR, reverse transcription-polymerase chain reaction; n.d., not determined.

Table II. Association between Tax-specific CTL counts and other pathological findings.

\section{Tax-CTL}

\begin{tabular}{lcc} 
Characteristics & Mean $\left(\right.$ cells $\left./ \mathrm{mm}^{2}\right)$ & P-value \\
\hline Foxp3 expression & & \\
Foxp3 positive rate $>30 \%$ & 0.0 & 0.0006 \\
Foxp3 positive rate $<30 \%$ & 4.6 & \\
HLA-A24 expression & & \\
HLA-A24 positive rate $>30 \%$ & 3.4 & 0.7000 \\
HLA-A24 positive rate $<30 \%$ & 3.0 & \\
Tax expression & & \\
Tax positive rate $>10 \%$ & 2.8 & \\
Tax positive rate $<10 \%$ & 3.6 & \\
Morphology & & \\
Medium cell predominant & 2.0 & \\
Large cell predominant & 4.0 & \\
Anaplastic variant & 5.0 & \\
\hline
\end{tabular}

Foxp3, forkhead box P3; HLA, human leukocyte antigen; CTL, cytotoxic T lymphocyte.

that only CD8/multimer double-positive cells (Fig. 2) were regarded as a positive signal.

Foxp3 immunostaining was also analyzed. Foxp3 expression $>30 \%$ was defined as positive, according to previous reports (6). Among the 15 cases, 5 (33\%) were positive for Foxp3 (Fig. 1 and Table I). Of the Foxp3+ cases, 4 were included in the pleomorphic medium type, accounting for $80 \%$, a significantly higher percentage than that observed for the other two types.

There was an inverse correlation between Tax-specific CTL expression and Foxp3 expression (Table II).

Tax-specific CTL function in lymph nodes of ATLL patients. To reveal whether Tax-specific CTLs were activated or not, immunostaining for INF $\gamma$ was performed. The number of INF $\gamma$-positive cells ranged from 0.0 to 3.5 cells $/ \mathrm{mm}^{2}$, and was lower than the number of Tax-specific CTLs in the same area, with certain Tax-specific CTLs being inactive (Fig. 3 and Table I).

Tax and HLA-A24 expression in ATLL cells. In the present study, HLA-A24-positive ATLL patients were selected by a PCR method. RT-PCR products for Tax were detected in 14/14 patients examined (Fig. 4).

Immunohistochemical staining of Tax protein revealed that the number of positive lymphoma cells ranged from 5 to $18 \%$, and the number of HLA-A24-positive lymphoma cells ranged from 1 to $95 \%$ (Fig. 3 and Table I). There was no correlation between the number of Tax-specific CTLs and positivity for Tax or HLA-A24 (Table II).

\section{Discussion}

The present study suggested that lymphoma cells in ATLL patients may evade CTL-mediated immune control by expression of Foxp3. 

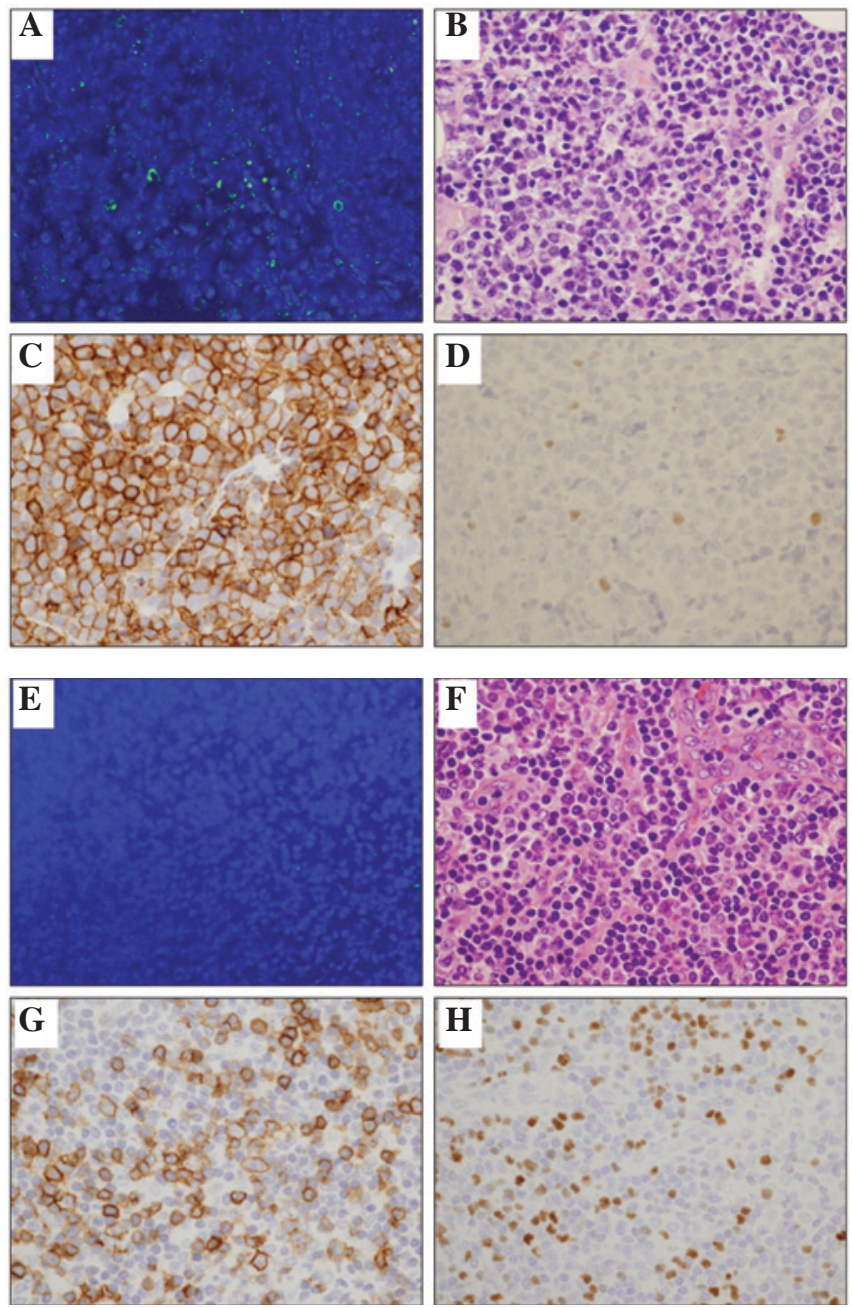

Figure 1. In situ detection of Tax-specific CTLs and immunostaining for Foxp3. Case 2: (A) Tax-specific CTL: Positive. (B) HE: Large cell variant (C) CD45RO: Positive, (D) Foxp3 positive rate: 2\%. Case 6: (E) Tax-specific CTL: Negative. (F) HE: Small cell variant. (G) CD45RO: Positive. (H) Foxp3 positive rate: $40 \%$. Magnification, $x 400$. CTL, cytotoxic T lymphocyte Foxp3, forkhead box P3; HE, hematoxylin and eosin; CD, cluster of differentiation.

In previous reports, it was controversial whether ATLL cells function as Treg cells $(7,20,21)$. There were three types of opinions about this argument: First, ATLL cells have a Treg function (7). In that study, the authors separated CD4+ C-C motif chemokine receptor (CCR) 4+ ATLL cells using biotin-conjugated KM2160 (monoclonal antibodies to CCR4) and Anti-Botin MicroBeads. The proliferation of CD4+CCR4+ ATLL cells and CD4+ non-ATLL cells, as well as their IFN- $\gamma$ production in response to T-cell receptor (TCR) stimulation in the presence of autologous adenomatous polyposis coli, was assessed. The results demonstrated that CD4+CCR4+ ATLL cells from a subset of patients were able to suppress the proliferation and IFN $\gamma$ production of autologous CD4+non-ATLL cells in vitro at a ratio of 1:1. Second, ATLL cells do not have a Treg function (20). In that study, the regulatory function of ATL cells was evaluated using an alloimmune mixed lymphocyte reaction system. CD4+CD25+ T-cells and CD8+ T-cells were isolated from peripheral blood mononuclear cells (PBMCs) of ATL patients and a normal healthy donor with immunomagnetic beads. In the healthy control, CD4+CD25+
T-cells inhibited the proliferation of autologous CD8+ T-cells, thus displaying the ordinary suppressive activity of Treg cells. However, in the ATL patients tested, the proliferation of CD8+ T-cells was not suppressed by CD4+CD25+ T-cells. In addition to circulating ATL cells, single-cell suspensions of skin-infiltrating tumor cells did not inhibit autologous CD8+ T-cell proliferation at any ratio. Third, not ATLL cells but only CD4+Foxp3+ cells increasing in peripheral blood of ATLL patients have a suppressor function (21). In that study, the authors positively selected CD4+ cells from fresh PBMCs of patients with ATLL, and then used the respective TCR V $\beta$-specific monoclonal antibodies to isolate the expanded T-cell clones. Next, the authors purified the CD25+ cells from the remaining (TCR V $\beta+$-depleted) population, and then added three cell populations (CD4+TCR V $\beta+, \mathrm{CD} 4+\mathrm{CD} 25+$ and CD4+TCR V $\beta-C D 25-)$ to $C D 4+C D 25-$ cells labeled with carboxyfluorescein succinimidyl ester (CFSE) from a healthy individual. The authors investigated the frequency of CFSE expression in CD4+ cells after incubation for 4 days in order to assess the inhibition of proliferation of $\mathrm{CD} 4+\mathrm{CD} 25$ - cells. It was observed that only the CD4+CD25+ population caused strong inhibition of proliferation, and that this $\mathrm{CD} 25+$ population expressed high frequency of Foxp3.

In these studies, peripheral blood cells were separated using the autoMACS ${ }^{\circledR}$ magnetic separation system. In order to separate ATLL cells, the authors used Tax, Foxp3, CD4, CD25 and CCR4 as markers. However, it remained difficult to distinguish ATLL cells from normal Treg cells phenotypically and to separate the population physically, since both cell types express cell-surface markers characteristic of Treg cells (6).

In our study, it was concluded that ATLL cells, which were positive for Foxp3, had a Treg-like function, since morphologically atypical T-cells were positive for Foxp3, and that there was an inverse correlation between Foxp3 expression and Tax-specific CTL counts.

Karube et al reported that ATLL cells were positive for Foxp3, and that there was a correlation between Foxp3 expression and Epstein-Barr virus (EBV)-positive cell counts (6). This phenomenon may occur by the same mechanism, since Foxp3+ ATLL cells may suppress EBV-specific CTLs.

Treg cells are known to have numerous suppressive mechanisms, including suppressive cytokines, metabolic disruption and targeting of dendritic cells (11). Treg cells secrete perforin and granzyme B, and induce effector T-cell apoptosis, being CTLs one of their targets (11). In addition, Treg cells secrete transforming growth factor- $\beta 1$ and change active CTLs to inactive ones (11). In our study, the number of INF $\gamma$-positive cells was lower than the number of Tax-specific CTLs in the same area, and it was concluded that several Tax-specific CTLs may be inactive due to the T-reg-like function of ATLL cells. Harashima et al (26) investigated cellular immune responses of ATL patients who obtained complete remission following non-myeloablative allogenic peripheral blood HSCT using cultured PBMCs from patients and healthy donors. The authors measured the cytotoxic activity of CTLs by 6-h ${ }^{51} \mathrm{Cr}$ release assay at various effector-to-target cell ratios (27). INF- $\gamma$ production of effector cells was also measured, and the results indicated that Tax-specific CTLs in ATLL patients are inactive, and that the Tax-specific CTL response is strongly activated following HSCT in certain ATLL patients 

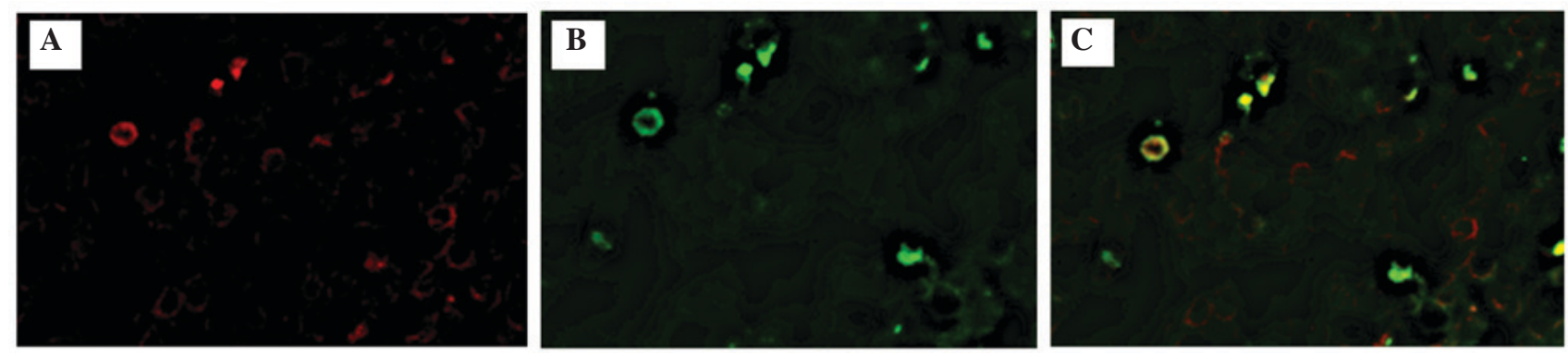

Figure 2. In situ detection of Tax-specific cytotoxic T lymphocytes (case 2). Confocal laser scanning microscopy with (A) anti-cluster of differentiation 8 antibody (red channel) and (B) fluorescein isothiocyanate-conjugated multimetric human leukocyte antigen-A24/Tax construct (green channel) in lymph nodes of an adult T-cell leukemia/lymphoma patient. (C) Double positive cells appear yellow. Magnification, x400.
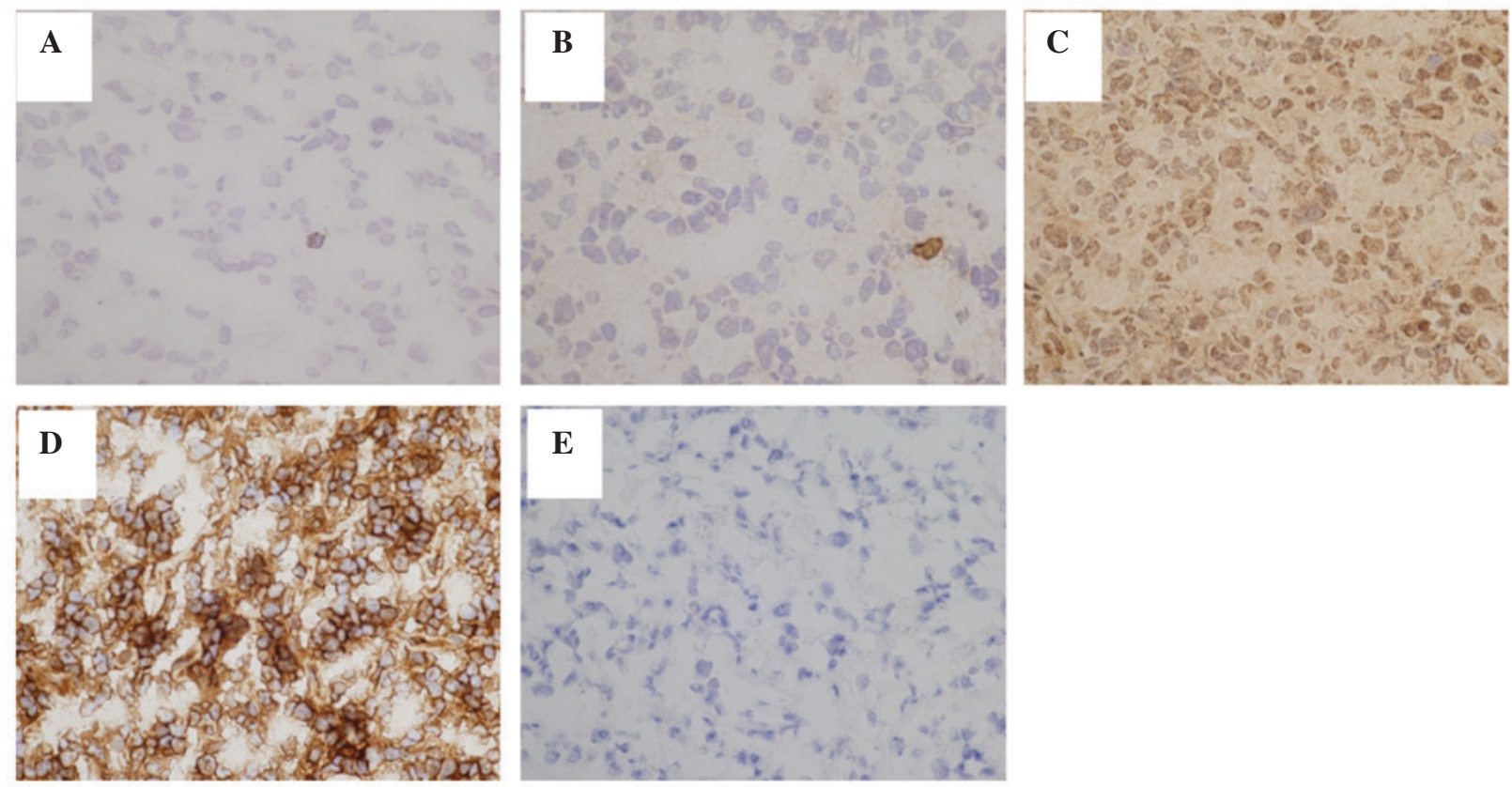

Figure 3. Immunostaining for INF $\gamma$, Tax and HLA-A24. (A) There was 1 INF $\gamma$-positive cell/ $/ \mathrm{mm}^{2}$ (Case 9). (B) Tax exhibited $2 \%$ positive lymphoma cells (Case 9). (C) Tax displayed 18\% positive lymphoma cells (Case 3). (D) HLA-A24 was positive in 95\% of lymphoma cells (Case 1). (E) Lymphoma cells were negative for HLA-A24 expression (Case 3). Magnifcation, x400. HLA, human leukocyte antigen; INF, interferon.

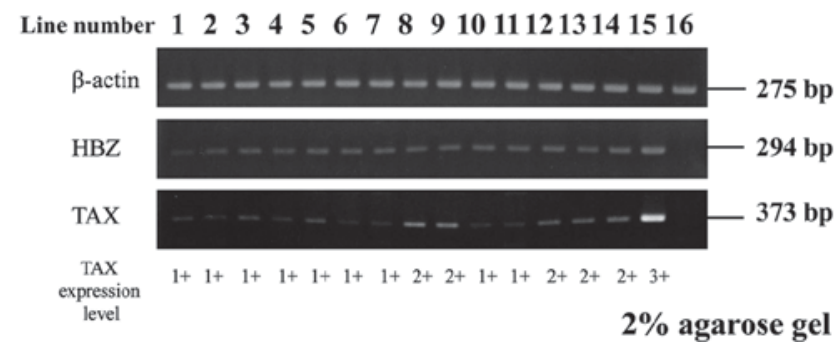

Figure 4. Messenger RNA expression of Tax and HBZ, as evaluated by RT-PCR. RT-PCR products for Tax and HBZ were detected in 14/14 patients. RNA could not be obtained for Case 2. Lines 1-14 represent cases 1 and cases 3-15, in chronological order. ML4 cells, Line 15; Jurkat cells, Line 15. RT-PCR, reverse transcription-polymerase chain reaction; HBZ, HTLV-1 basic leucine zipper factor.

in long-term remission, by using peripheral blood of ATLL patients.

It has been reported that the level of Tax expression in HTLV-1-infected cells decreases during disease progression, and Tax transcripts have been detected only in $\sim 40 \%$ of established ATLL cases in peripheral blood (18).

In our study, immunohistochemical staining of Tax protein revealed the number of positive cells to range from 5 to $18 \%$, and RT-PCR products for Tax were detected in all patients. Since the results of RT-PCR may be influenced not only by the presence of ATLL cells, but also by the presence of HTLV-1-infected cells, immunohistochemistry was performed. The results of immunostaining demonstrated that few ATLL cells and few HTLV-1-infected lymphocytes were positive for Tax in certain cases. Thus, even if Tax expression is decreased, Tax protein can be a target of Tax-specific CTLs.

As aforementioned, Tax-specific CTLs were considered to be important in controlling ATLL. Recently, a humanized anti-CCR4 antibody, mogamulizumab, yielded a good response in CCR4-positive ATLL patients (28). While mogamulizumab attacks CCR4-positive tumor cells, it also attacks Treg cells (29). Therefore, CTLs are activated (29). Furthermore, immunotherapy with anti programmed-death (PDL) 1 antibody and anti CTL antigen (CTLA)-4 antibody has become an 
increasingly appealing therapeutic strategy for patients with cancer, with numerous late-stage clinical trials demonstrating overall survival advantages in melanoma and castration-resistant prostate cancer $(30,31)$. CTLA-4 and PDL1 have distinct roles in regulating immunity, and blocking these pathways resulted in the removal of inhibitory signals and activation of CTLs $(32,33)$. These immunotherapies may become a promising strategy for ATLL in the future.

In humans, CTLs are important in controlling virus-infected cells and malignant cell growth (34). The interactions between CTLs and their target cells are mediated by HLA class I antigens loaded with viral and tumor antigen-derived peptides, along with co-stimulatory receptor/ligand stimuli (34).

The present finding that HLA-A24-positive lymphoma cells ranged from 1 to $95 \%$ is in agreement with the findings of previous studies on ATLL, which reported that the expression of surface MHC-I was significantly downregulated in the resistant cells (27). In order to escape from CTL recognition, viruses and tumor cells have developed strategies to inhibit the expression and/or function of HLA class I antigens (19). For example, adenovirus, EBV, human papillomavirus, human immunodeficiency virus and human herpes virus have such strategies (35). HTLV-1 also has such a strategy. HTLV-1 inhibits the association of MHC class I heavy chain with $\beta 2$ microglobulin and inhibits the expression of HLA class I antigens (36). In malignant tumors such as squamous cell carcinoma, small cell carcinoma, colon cancer, cutaneous melanoma, prostate cancer and Hodgkin lymphoma, HLA class I antigen downregulation or loss has been detected (19). The region of chromosome 6 , which carries the human MHC (37), is unstable during malignant transformation of cells (38). Mutations may lead to downregulation or loss of the HLA class I antigen-tumor associated-derived peptide complex (38), and the same mechanism may be present in ATLL patients.

In conclusion, our study demonstrated that lymphoma cells in ATLL patients evaded CTL-mediated immune control by expression of Foxp3 and downregulation of Tax and HLA-A24. To the best of our knowledge, this is the first experiment that revealed how Tax-specific CTLs behave in lymph nodes of ATLL patients, and supported previous cell culture experiments that reported that the Tax-specific CTL response was important to control HTLV-1 infection. This finding suggest that reactivation of the Tax-specific CTL response may provide prophylactic and therapeutic approaches for HTLV-1 carriers and for ATLL patients whose ATLL cells retain the ability to express Tax.

\section{Acknowledgements}

The present study was supported by a Grant-in-Aid for Scientific Research of The Ministry of Education, Culture, Sports, Science and Technology (Tokyo, Japan; grant no., 26460446).

\section{References}

1. Poiesz BJ, Ruscetti FW, Gazdar AF, Bunn PA, Minna JD and Gallo RC: Detection and isolation of type $\mathrm{C}$ retrovirus particles from fresh and cultured lymphocytes of a patient with cutaneous T-cell lymphoma. Proc Natl Acad Sci USA 77: 7415-7419, 1980.

2. Verdonck K, González E, Van Dooren S, Vandamme AM, Vanham G and Gotuzzo E: Human T-lymphotropic virus 1: Recent knowledge about an ancient infection. Lancet Infect Dis 7: 266-281, 2007.
3. Gessain A: Epidemiology of HTLV-1 and associated disease. In: Human T-cell Lymphotropic Virus Type-1. Hollsberg P and Hafler DA (eds). Wiley, Chichister 33-64, 1996.

4. Tobinai K:Currentmanagement of adultT-cell leukemia/lymphoma. Oncology (Williston Park) 23: 1250-1256, 2009.

5. Swerdlow SH, Campo E, Harris NL, Jaffe ES, Pileri SA, Stein H, Thiele J and Vardiman JW (eds): WHO Classification of Tumours of Haematopoietic and Lymphoid Tissues. Vol 2.4th edition. IARC Press, Lyon, France, 2008.

6. Karube K, Aoki R, Sugita Y, Yoshida S, Nomura Y, Shimizu K, Kimura Y, Hashikawa K, Takeshita M, Suzumiya J, et al: The relationship of Foxp3 expression and clinicopathological characteristics in adult T-cell leukemia/lymphoma. Mod Pathol 21: 617-625, 2008.

7. Yano H, Ishida T, Inagaki A, Ishii T, Kusumoto S, Komatsu H, Iida S, Utsunomiya A and Ueda R: Regulatory T-cell function of adult T-cell leukemia/lymphoma cells. Int J Cancer 120: 2052-2057, 2007.

8. Marsh BJ: Infectious complication of Human T cell leukemia/lymphoma virus type I infection. Clin Infect Dis 23: 138-145, 1996.

9. Montes M, Sanchez C, Verdonck K, Lake JE, Gonzalez E, Lopez G, Terashima A, Nolan T, Lewis DE, Gotuzzo E and White AC Jr. Regulatory T cell expansion in HTLV-1 and strongyloidiasis co-infection is associated with reduced IL-5 responses to strongyloides stercoralis antigen. PLoS Negl Trop Dis 3: e456, 2009.

10. Marcos LA, Terashima A, Dupont HL and Gotuzzo E: Strongyloides hyperinfection syndrome: An emerging global infectious disease. Trans R Soc Trop Med Hyg 2: 314-318, 2008.

11. Sakaguchi S, Wing K, Onishi Y, Prieto-Martin P and Yamaguchi T: Regulatory T cells: How do they suppress immune responses? Int Immunol 21: 1105-1111, 2009.

12. Suzuki S, Masaki A, Ishida T, Ito A, Mori F, Sato F, Narita T, Ri M, Kusumoto S, Komatsu H, et al: Tax is a potential molecular target for immunotherapy of adult T-cell leukemia/lymphoma. Cancer Sci 3: 1764-1773, 2012.

13. Seiki M, Hattori S, Hirayama Y and Yoshida M: Human adult T-cell leukemia virus: Complete nucleotide sequence of the provirus genome integrated in leukemia cell DNA. Proc Natl Acad Sci USA 80: 3618-3622, 1983.

14. Franchini G: Molecular mechanisms of human T-cell leukemia/lymphotropic virus type I infection. Blood 86: 3619-3639, 1995.

15. Jin DY, Spencer F and Jeang KT: Human T cell leukemia virus type 1 oncoprotein Tax targets the human mitotic checkpoint protein MAD1. Cell 93: 81-91, 1998.

16. Grossman WJ, Kimata JT, Wong FH, Zutter M, Ley TJ and Ratner L: Development of leukemia in mice transgenic for the Tax gene of human T-cell leukemia virus type I. Proc Natl Acad Sci USA 92: 1057-1061, 1995.

17. Kannagi M: Immunologic control of human T-Cell leukemia virus type I and adult T-cell leukemia. Int J Hematol 86: 113-117, 2007.

18. Takeda S, Maeda M, Morikawa S, Taniguchi Y, Yasunaga J, Nosaka K, Tanaka Y and Matsuoka M: Genetic and epigenetic inactivation of Tax gene in adult T-cell leukemia cells. Int $\mathbf{J}$ Cancer 109: 559-567, 2004.

19. Seliger B, Cabrera T, Garrido F and Ferrone S: HLA class I antigen abnormalities and immune escape by malignant cells. Semin Cancer Biol 12: 3-13, 2002.

20. Shimauchi T, Kabashima K and Tokura Y: Adult T-cell leukemia/lymphoma cells from blood and skin tumors express cytotoxic T lymphocyte-associated antigen- 4 and Foxp3 but lack suppressor activity toward autologous CD8+ T cells. Cancer Sci 9: 98-106, 2008.

21. Toulza F, Nosaka K, Takiguchi M,Pagliuca T, Mitsuya H, Tanaka Y, Taylor GP and Bangham CR: Foxp3+ regulatory T cells are distinct from leukemia cells in HTLV-1-associated adult T-cell leukemia. Int J Cancer 5: 2375-2382, 2009.

22. Little AM and Parham P: Polymorphism and evolution of HLA class I and II genes and molecules. Rev Immunogenet 1: 105-123, 1999.

23. Satou Y, Yasunaga J, Yoshida M and Matsuoka M: HTLV-1 basic leucine zipper factor gene mRNA supports proliferation of adult $\mathrm{T}$ cell leukemia cells. Proc Natl Acad Sci USA 103: 720-725, 2006.

24. Andersen MH, Pedersen LO, Capeller B, Bröcker EB, Becker JC and thor Straten P: Spontaneous cytotoxic T-cell responses against survivin-derived MHC class I-restricted T-cell epitopes in situ as well as ex vivo in cancer patients. Cancer Res 16: 5964-5968, 2001.

25. Schrama D, Pedersen L, , Keikavoussi P, Andersen MH, Straten Pt Pt, Bröcker EB, Kämpgen E and Becker JC: Aggregation of antigen-specific $\mathrm{T}$ cells at the inoculation site of mature dendritic cells. J Invest Dermatol 9: 1443-1448, 2002. 
26. Harashima N, Kurihara K, Utsunomiya A, Tanosaki R, Hanabuchi S, Masuda M, Ohashi T, Fukui F, Hasegawa A, Masuda T, et al: Graft-versus-Tax response in adult T-cell leukemia patients after hematopoietic stem cell transplantation. Cancer Res 64: 391-399, 2004.

27. Ohashi T, Hanabuchi S, Suzuki R, Kato H, Masuda T and Kannagi M: Correlation of major histocompatibility complex class I downregulation with resistance of human T-cell leukemia virus type 1-infected T cells to cytotoxic T-lymphocyte killing in a rat model. J Virol 76: 7010-7019, 2002.

28. Yamamoto K, Utsunomiya A, Tobinai K, Tsukasaki K, Uike N, Uozumi K, Yamaguchi K, Yamada Y, Hanada S, Tamura K, et al: Phase I study of KW-0761, a defucosylated humanized anti-CCR4 antibody, in relapsed patients with adult T-cell leukemia-lymphoma and peripheral T-cell lymphoma. J Clin Oncol 28: 1591-1598, 2010.

29. Tobinai K, Takahashi T and Akinaga S: Targeting chemokine receptor CCR4 in adult T-cell leukemia/lymphoma and other T-cell lymphomas. Curr Hematol Malig Rep 7: 235-240, 2012.

30. Kono K: Current status of cancer immunotherapy. J Stem Cells Regen Med 30: 8-13, 2014.

31. Tosti G, Cocorocchio E and Pennacchioli E: Anti-cytotoxic T lymphocyte antigen-4 antibodies in melanoma. Clin Cosmet Investig Dermatol 6: 245-256, 2013.
32. Chambers CA, Kuhns MS, Egen JG and Allison JP: CTLA-4-mediated inhibition in regulation of T cell responses: mechanisms and manipulation in tumor immunotherapy. Annu Rev Immunol 19: 565-594, 2001.

33. Parekh VV, Lalani S, Kim S, Halder R, Azuma M, Yagita H, Kumar V, Wu L and Kaer LV: PD-1/PD-L blockade prevents anergy induction and enhances the anti-tumor activities of glycolipid-activated invariant NKT cells. J Immunol 182: 2816-2826, 2009.

34. Andersen MH, Schrama D, Thor Straten P and Becker JC: Cytotoxic T cells. J Invest Dermatol 126: 32-41, 2006.

35. Seliger B, Ritz U and Ferrone S: Molecular mechanisms of HLA class I antigen abnormalities following viral infection and transformation. Int J Cancer 118: 129-138, 2006.

36. Johnson JM, Nicot C, Fullen J, Ciminale V, Casareto L, Mulloy JC, Jacobson S and Franchini G: Free major histocompatibility complex class I heavy chain is preferentially targeted for degradation by human T-cell leukemia/lymphotropic virus type 1 p12I protein. J Virol 75: 6086-6094, 2001.

37. Francke U and Pellegrino MA: Assignment of the major histocompatibility complex to aregion of the short arm of human chromosome 6. Proc Natl Acad Sci USA 74: 1147-1151, 1977.

38. Richardson JH, Edwards AJ, Cruickshank JK, Rudge P and Dalgleish AG: In vivo cellular tropism of human T-cell leukemia virus type 1. J Virol 64: 5682-5687, 1990. 